\title{
Ontology for flood management: a proposal
}

\author{
D. De Wrachien ${ }^{1}$, J. Garrido ${ }^{2}, \mathrm{~S}$. Mambretti $^{3} \&$ I. Requena ${ }^{2}$ \\ ${ }^{1}$ Department. of Agricultural Engineering, \\ State University of Milan, Italy \\ ${ }^{2}$ Department of Computer Science and Artificial Intelligence, \\ University of Granada, Spain \\ ${ }^{3}$ DIIAR, Politecnico di Milano, Italy
}

\section{Abstract}

Floods are very complex phenomena involving a large number of players. In this context not only the hydrologic and hydraulic studies are required, but also those related to vulnerability assessment, town and emergency planning as well as all the matters related to the economic issues: damage, insurance and the psychological diseases which can arise after flood events.

Thus, people with widely different roles, backgrounds, and profiles are in charge of each phase of a large number of complex procedures. Not surprisingly, communication problems often arise because different names may be used to refer to the same concepts. Moreover, relevant information can vary, depending on the user.

Effective communication is essential since the results produced by a person in a certain phase of the process can be the input that another person needs in the next phase, i.e. raw data versus elaborated data, forecasting versus impact evaluation, level of detail, hazard assessment, vulnerability assessment, economic evaluation, environmental assessment, etc.

Ontologies, which can be defined as an explicit representation of a conceptualization, are tools that can be used to solve such very complex and interdisciplinary problems because they specify a conceptual framework or terminology.

The paper introduces an ontology for floods. This ontology is developed using the existing Environmental Impact Assessment (EIA) ontology and it is represented with OWL-DL (Web Ontology Language - Description Logics).

Among the different causes for flood, only those related to river overflows are taken into account in the ontology so far. It is shown as ontologies are useful 
because they represent the most meaningful knowledge associated with a problem. Moreover, they are also useful because they permit reasoning and inference processes. Inference mechanisms make recommendation procedures possible.

Keywords: hazards, floods, knowledge representation, brief ontology.

\section{Introduction}

The terms "flood" and "flooding" are often used in different ways. According to International Commission on Irrigation and Drainage (ICID) [1] flood is "a temporary condition of surface water (river, lake, sea) in which the water level and/or discharge exceed a certain value, thereby escaping their normal confines". Flooding is defined "as the overflowing or failing of the normal confines of a river, stream, lake, canal, sea or accumulation of water as a result of heavy precipitation where drains are lacking or their discharge capacity is exceeded".

Although flooding is a serious hazard in humid regions, it can be devastating also in semiarid regions, where high rates of runoff following storms produce widespread flood damage down valley. Recurring floods are also typical in coastal and estuarine zones [2].

To cope with these hazards, it is imperative that human society adopts an effective flood hazard management approach which has to be in harmonious coexistence with floods. In practical terms, the chance of flooding can never be eliminated entirely. However, the consequences of flooding can be mitigated by appropriate behaviour and actions. To be effective, the hazard approach must be embodied in the broader context of integrated catchment planning, and flood must be regarded as one of the many issues involved in the appropriate management of a catchment [3].

People with widely different roles, backgrounds, and profiles are in charge of these different issues and therefore communication problems may arise because of the different definitions apply to the same concepts. Moreover, relevant information can vary, depending on the user, and the results produced by a person in a certain phase of the process are the input that another person needs in the next phase.

Ontologies are a tool that can be used to solve such problems because they specify a conceptual framework or terminology.

Although there are a wide variety of definitions of ontology, many of them are too focused on a specific application or require a background in logic. However, according to Gruber [4] "a body of formally represented knowledge is based on a conceptualization: the objects, concepts, and other entities that are assumed to exist in some area of interest and the relationships that hold among them". This Author goes on to define ontology as an explicit representation of a conceptualization. This definition was considered sufficient for the purposes of our study.

Ontologies also provide explicit definitions and restrictions for concepts, i.e. Knowledge Representation (KR). They can be used to describe a context or the domain of a context [5]. The Environmental Assessment Ontology [6] provides a 
valid conceptual framework for modelling flood-related knowledge. The paper shows the inclusion of a semantic model for flood management in the EIA ontology and the generation of a brief ontology in order to simplify and optimize the use of the ontology for flood information. It describes the semantic modelling of floods and flood-related concepts [7, 8].

\section{Flood management}

Risk is an integral part of social and economic processes and is often increased by human interference with natural hydro-meteorological phenomena. The struggle against extreme events like floods and droughts is as old as mankind. In the last decades, new challenges are likely to influence risk management measures and policies:

- $\quad$ increasing world population and economic growth lead to a more intense use of water and land resources;

- there is a rising awareness of the need of integrated flood risk management, considering the river basin as the basic planning unit;

- due to the relentless urbanisation process, at worldwide level, hazards are increasingly transforming into disasters putting development at risk;

- $\quad$ in flood prone areas there may be the problem of land subsidence. This problem makes such areas increasingly vulnerable;

- climate change is likely to impact climate variability, making extreme events more severe and more frequent even if, compared to the items listed above, impacts of climate change are of limited importance;

- there is a rising concern that damages resulting from water related disasters are growing disproportionately worldwide.

To cope with these challenges involves taking decisions and actions about appropriate levels of risks. These decisions and actions may be divided into risk analysis procedures and risk management cycle.

In recent years risk analysis has emerged as one of the most appropriate methods nowadays available to assess natural hazards, like floods and other water related disasters. This methodology proved to be comparatively reliable in determining the hazard potential and the related probability of occurrence of defined extreme events, but this process requires expert knowledge to identify the potential risks and then to estimate the likelihood and the impacts of these risks. Risk likelihood and risk impact comprise uncertainty. In this context, the last two decades have observed an amazing progress in the use of probability theory to assess uncertainty. However, floods are natural hazards, which occur periodically and episodically and cannot be prevented.

Therefore, the responsible authorities in most European countries developed methods of integrated risk management, which follow, mainly, the approach to express risk as a product of hazard and values at risks. To this end, it is worth noting that, whilst in the past the concept of risk was primarily intended as a measure of the probability of a system's failure, it has got, nowadays a more complex meaning. The risk has to be considered as a combination of both the probability and the magnitude of the consequences of a system's failure, and so, 
as the mathematical expectation of the consequence, taking into account of all significant hazards and all significant mechanisms of failure [9].

With regards to the risk management, according to the International Strategy for Disaster Reduction (ISDR) [10], its cycle comprises "the systematic process, administrative decisions, organisation, operational skills and abilities to implement policies, strategies and coping capacities of the society and communities to lessen the impact of natural hazards and related environmental and technological disasters". This covers all forms of activities, including structural and non - structural measures to avoid or to limit adverse effects of hazards.

On the whole, the risk management approach consists of systemic actions set up in a cycle of preparedness, response and recovery that should form part of any integrated flood risk management.

Preparedness consists of preventive and precautionary measures to prepare for an event before it occurs. It aims at minimizing the effect of development activities on accentuating the magnitude of hazards, reducing the exposure to natural hazards and minimising the socio - economic vulnerability of people and material assets exposed to these hazards. Response consists of measures that limit the effects of exposure to a hazard and its duration. It mainly focuses on alerting potentially affected people, rescuing victims and providing assistance in case of need. The recovery phase aims at enabling the economic and social activities to return to normal with a minimum delay. It also involves the analysis of the disaster in order to learn lessons and integrate corrective measures into prevention and preparedness plans. To this end, it is important to underline that the effectiveness of the risk management cycle in reducing risks and damages depends, also, on the political will to apply the risk management principles in developing planning, the existence of well defined institutional responsibilities and on a democratic process of consultation and social control with effective governance.

The challenge before the international community is to support these activities, particularly in least developed countries, where resources are limited, by means of actions aiming at:

- $\quad$ informing policy makers and the public of the trends in water - related risks and policy options to mitigate those risks;

- introducing long-term land use planning, taking into account the concerned aspects of flood risk management (including risk assessments) and adaptive management to reduce vulnerability to risks, that may be increase in time, due to the processes as described before;

- raising awareness of water-related hazards and improving the capacity of communities to respond effectively;

- developing conventional and state-of-the-art technologies and monitoring systems tailored to local conditions for water-hazard alerts;

- fostering specific capacity development programmes for water managers.

Finally, it is known as many players are involved in flood management, each of them having different backgrounds, skills and expectations. There is a need 
for a planning framework able to explain all the relations between the players and the role of each of them.

\section{Ontologies}

Depending on the application and context where the ontologies are used, they provide a mechanism to share knowledge by using a common vocabulary; they allow semantic labelling, exchanging and reusing knowledge; they can be also used to establish a communication protocol. Its formal background makes possible the semantic, logic and formal descriptions without additional tools. Its use in genetics has spread to fields such as knowledge engineering, artificial intelligence and computer science, language processing, knowledge representation, cooperative information systems, ecommerce, bioinformatics, database design and integration, smart integration of information, information retrieval and knowledge management.

Roughly speaking, an ontology consists of properties, classes, instances and operators:

- Classes correspond to concepts or elements in the real world.

- Properties are relationships whose domain and range are defined in terms of concepts.

- Instances are individuals of classes.

- Operators represent the allowed symbols which provide the set of operations or mechanisms for the concept definitions.

The concepts can be formally defined by axioms using the operators, properties, instances and other concepts. For example, a concept can be defined as the union of other concepts.

The concepts' definitions are supported by the Description Logic (DL), which are a family of knowledge representation languages. The set of operators and its use implies different levels of complexity and efficiency for the description logic. In general, lower expressiveness of the language implies higher efficiency but poorer definitions whereas higher expressiveness brings more flexibility in the definitions but decidability problems in the worse scenario i.e. it is not guaranteed to find an answer for a question in a finite amount of time.

Although there are several languages to represent ontologies, OWL (Web Ontology Language) is the current standard according to the W3C (World Wide Web Consortium). The $\mathrm{W} 3 \mathrm{C}$ is an international community that develops open standards to ensure the long-term growth of the Web. The set of operators in OWL consists in union, intersection, negation, one-of, cardinality, universal and existential operator.

Beyond the concept definition, there is a structural relationship which allows representing the concepts as taxonomy. This relationship is referred as IsA and provides a hierarchical representation of the concepts. Therefore, a concept will always have a parent and if two concepts are children of the same parent then they are called siblings. Additionally, if a concept $(X)$ is the parent of other concept $(Z)$ then the concept $Z$ is generalized or subsumed by the concept $X$ (commonly said that $\mathrm{Z}$ is a (IsA) $\mathrm{X}$ ). 
An ontology which only uses the IsA relationship is a taxonomy of concepts. However, we take advantage of ontologies by describing formal definitions because it allows implementing semantics and using reasoners. The reasoners are applications that check the consistency in the ontology i.e. there are no contradictions. They also make possible the inference mechanisms to discover new knowledge or launch queries over the ontology.

Let us suppose an ontology where the concepts Country and City are defined in form of classes. The individuals of these classes will be the instances of the concepts i.e. the current countries and their cities. If there is a property called hasCapital whose semantics implies that a country has a capital then the definition of the class Country could include a restriction to force that a Country must have a Capital. The example bellow shows how the countries Italy and France are related to their capitals.

- Class: City

- Class: Country ( $\exists$ hasCapital, ...)

- Instance of City: Rome

- Instance of City: Paris

- Instance of City: Barcelona

- Instance of Country: Italy (hasCapital Rome, ...)

- Instance of Country: France (hasCapital Paris, ...)

- Property: hasCapital (domain: Country, range: City)

Considering that all the cities are not capital, a concept Capital could be defined as anything that is capital of a country by defining the property isCapitalOf (domain: City, range: Country) as inverse of hasCapital: Capital ( $\exists$ isCapitalOf). If we do so, the instances Rome and Paris will be additionally inferred as individuals of the concept Capital.

There are many tutorials and literature about ontologies, for example, the tutorial for Protègè (an ontology editor [11]). Additionally, some books gather a list of applications based on ontologies and show how they are used [12]. Finally, as the complete ontology may be slow and difficult to deal with, because it includes a lot of knowledge that is not related directly to floods, brief ontologies may be built [13].

\section{Building an ontology for flood management}

An ontology can be used to model floods because of the close interrelation between the elements involved. Other approaches, such as databases, are not satisfactory since they only store data. However, our objective was to provide the representation of an entire system. This necessarily includes a specification of the connections between its components so that the model can perform reasoning tasks.

Although there is no standard for ontology development, there are principles and recommendations that can be followed. One of the most well-known set of 
guidelines is Methontology [14], which recommends reusing ontologies to the extent possible. Besides saving time and resources, this makes integration between applications considerably easier.

In particular, the methodology we have implemented is organized as follows:

1. Identification of the aim and the scope of the ontology: it can impose requirements in the construction phase.

2. The ontology construction process:

a. Getting knowledge: identification of concepts and relationships that should be in the ontology and design of a strategy for this task.

b. Encoding phase: defining classes, individuals and properties in OWL.

c. Integration with other ontologies in order to find an ontology with similar domain that can be reused or that can be specialized.

d. Inference in order to check consistency and correctness.

e. Creation of brief ontologies to extract relevant portions of the ontology for more specific domains.

3. Evaluation (by experts) and maintenance.

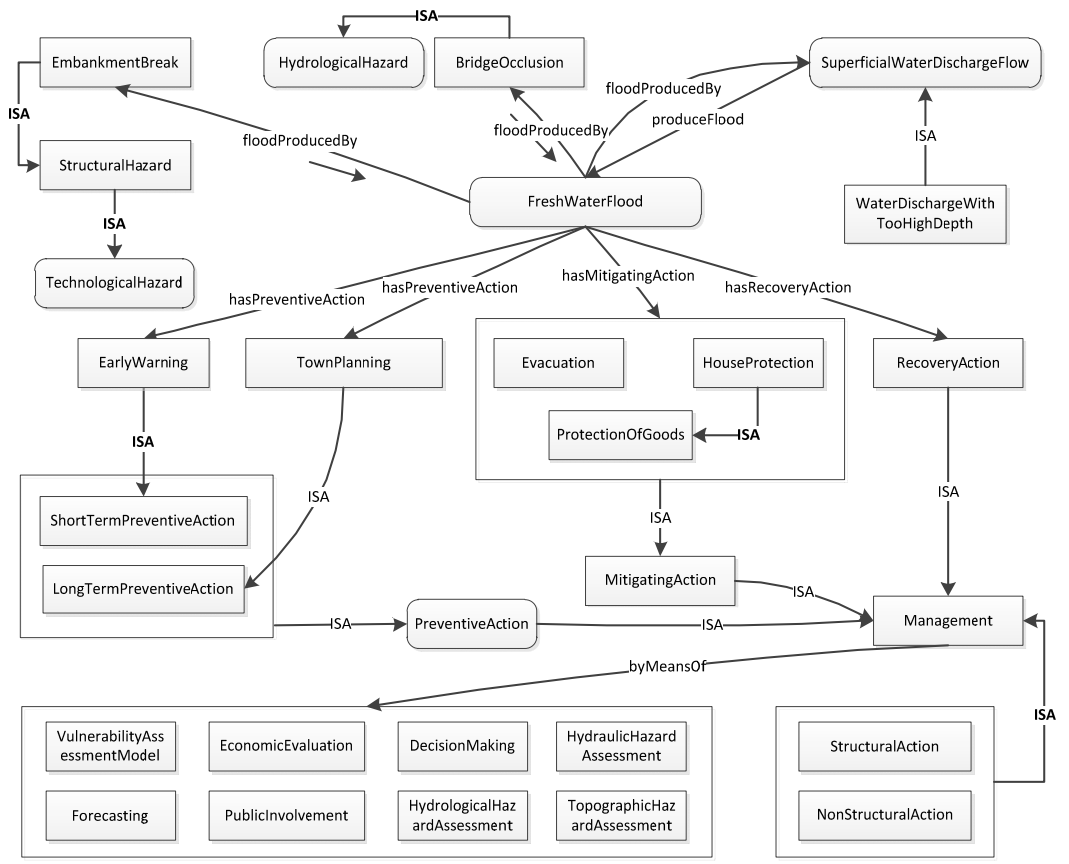

Figure 1: Conceptual schematization flood.

According to the previous methodology, the EIA ontology was chosen as a starting point for flood modelling because many of the concepts needed for such 
a representation are already in the EIA ontology. Some are directly included, whereas others are generalized in the representations of other concepts. In such cases, the concept can be included by adding more details to the existing taxonomy or by establishing new relationships to describe food-related knowledge. Alternately, new concepts can be added to the first level of the hierarchy.

In figure 1 the schematization of flood is shown. In the figure, nodes (rectangles) represent concepts, and they are linked by relations.

Flood is defined in the European Directive on assessment and management of flood risks as the temporary covering by water of land not normally covered by water. This general definition refers to all type of floods: river floods, flash floods, urban floods, and floods from the sea in coastal areas. Although all of these flood types share certain characteristics, this paper focuses on freshwater floods and particularly on river floods. For this reason, we have defined flood as a body of water that overflows its usual boundaries onto a land area with other land uses. This produces negative impacts caused by water velocity, depth, persistence, or any combination of the three.

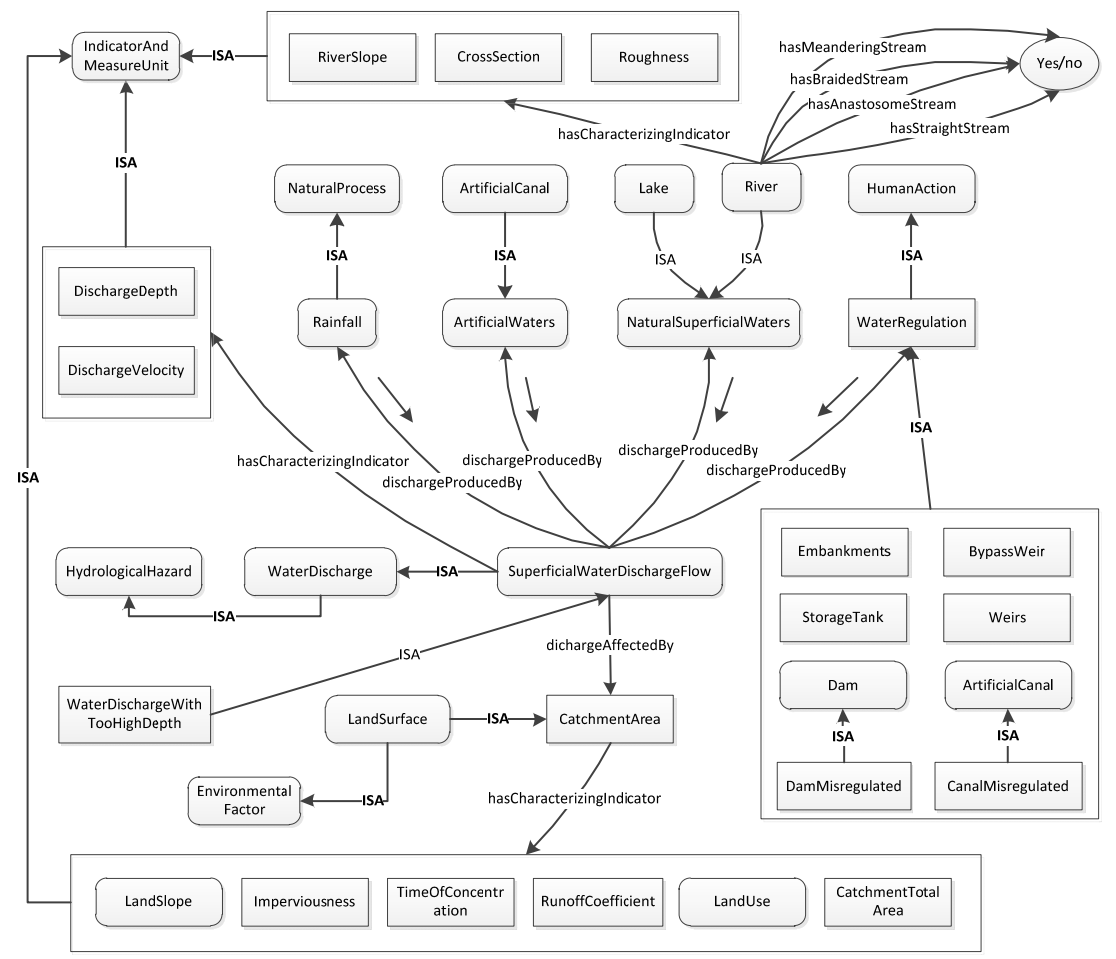

Figure 2: Conceptual schematization of superficial water discharge. 


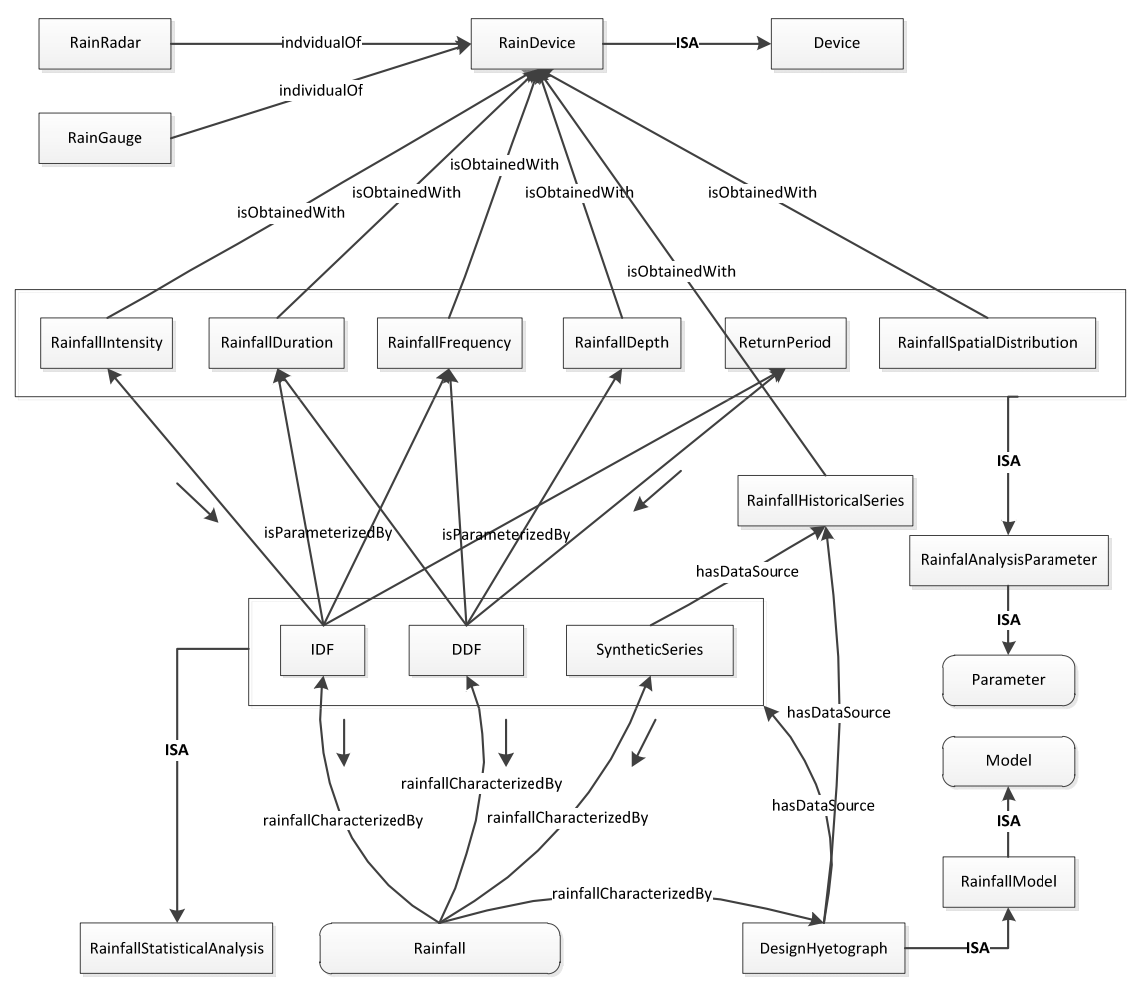

Figure 3: Conceptual schematization of rainfall.

Each of the concepts can be (and should be) expanded, as each of them is quite complex and includes a number of subconcepts and relations. As example, in figure 2 is reported a preliminary conceptual schematization of superficial water discharge flow, which appears in figure 1 as one of the causes of floods. Again, many events can cause a discharge in a river or in a channel; one of these is the rainfall, which is a concept that can be deepened and the result is shown in figure 3. Once this job is carried out for all concepts, and the relations between concepts are defined as well, a complete representation of the real world will be available.

\section{Concluding remarks}

Floods are complex phenomena involving a large number of players and therefore many studies are required, such as hydrologic and hydraulic, vulnerability assessment, town and emergency planning as well as all the matters related to the economic issues. Thus, people with widely different roles, backgrounds, and profiles are in charge of each phase of a large number of complex procedures and this can produce communication problems, especially because the concept of "relevant information" varies depending on the user. 
Moreover, the results produced by a person in a certain phase of the process can be the input that another person needs in the next phase.

Ontologies, which can be defined as an explicit representation of a conceptualization, are tools that can be used to solve such very complex and interdisciplinary problems because they specify a conceptual framework or terminology.

The paper introduces an ontology for floods. This ontology is developed using the existing Environmental Impact Assessment (EIA) ontology and it is represented with OWL-DL (Web Ontology Language - Description Logics). In the paper, first the conceptual framework for floods is shown. Here it is shown the reasons why floods may happen and how the different actions to be taken to reduce their consequences are related. Among the different causes for flood, only those related to river overflows are taken into account in the ontology so far. Then, as the river overflows are considered for floods, this concept has been expanded showing all subconcepts and properties related to it.

Finally, as an example, one of the concepts shown in the conceptual schematization of superficial water discharge (namely: rainfall) is expanded again in order to show the level of detail and deepness that can be reached in describing concepts. It is shown as ontologies are useful because they represent the most meaningful knowledge associated with a problem. Moreover, they are also useful because they permit reasoning and inference processes. Inference mechanisms make recommendation procedures possible.

\section{References}

[1] Schultz B. 2001: Irrigation, drainage and flood protection in a rapidly changing world. Irrigation and Drainage 50(4), pp. 261-277.

[2] Varnes D.J., and IAEG Commission on Landslides and other Mass Movements and landslide hazard zonation 1984: A review of principles and practice, 63 pp, UNESCO Press, Paris.

[3] Meadcroft I.C., Reeve D.E., Allop N.W.H., Diment R.P., Cross J. 1996: Development of new risk assessment procedures for coastal structures. Advances in Coastal Structures and Breakwaters, Thelford T. (Ed.), London, pp. 6-25.

[4] Gruber, T.R. 1993: Toward Principles for the Design of Ontologies Used for Knowledge Sharing Technical Report KSL 93-04 Knowledge Systems Laboratory Stanford University.

[5] Dey, A. \& Abowd, G. 2000: Towards a better understanding of context and context-awareness. Proceedings of the Workshop on the What, Who, Where, When and How of Context-Awareness (CHI), The Hague, Netherlands.

[6] Garrido, J. \& Requena, I. 2011: Proposal of ontology for environmental impact assessment. An application with knowledge mobilization. Expert System with Applications, 38 pp. 2462-2472.

[7] Garrido J., Requena I., Mambretti S. 2011: Building brief ontologies. A case study for floods management. Proceedings of the International 
Conference on Knowledge Engineering and Ontology Development, KEOD 2011, Paris, France, 26-29.

[8] Garrido J., Requena I., Mambretti S.: Semantic model for flood management Accepted for publication on the Journal of Hydroinformatics on 12th December 2011.

[9] Mambretti S., De Wrachien D., Sole A. 2008: Flood - risk assessment and hazard mitigation measures: Case studies and lessons learnt in Italy Proceedings of the International Conference on Flood Recovery Innovation and Response, Institute of Civil Engineers, London, United Kingdom.

[10] International Strategy for Disaster Reduction (ISDR) 2004: Terminology: Basin terms of disaster reduction. Updated version.

[11] Noy, N. F., McGuinness, D. L. 2005: Ontology Development 101: A Guide to Creating Your First Ontology. Stanford University, http://protege.stanford.edu

[12] Staab, S., Studer, R. 2004: Handbook on Ontologies. Springer

[13] Garrido J., Requena I. 2012: Towards summarising knowledge: Brief ontologies. Expert Systems with Application, V 39, 3, pp 3213-3222.

[14] Fernández, M., Gómez, A., Pazos, J. \& Pazos, A. 1999: Ontology of tasks and methods. IEEE Intelligent Systems and Their Applications, 14(1), pp. $37-46$. 